\title{
Ultrasound-Guided Intervention in a Sacroiliitis after Cancer Consequence or Coincidence? A Case Report and Review
}

\author{
Inês Ferro ${ }^{1 *}$, Pedro Carvalho Sá ${ }^{1}$, César Pires ${ }^{1}$, Filipe Carvalho ${ }^{2}$ and João Constantino ${ }^{2}$ \\ ${ }^{1}$ Resident in Physical and Rehabilitation Medicine, Centro de Medicina de Reabilitação da Região Centro, \\ Rovisco Pais, Portugal \\ ${ }^{2}$ Physical and Rehabilitation Medicine Specialist, Centro de Medicina de Reabilitação da Região Centro, \\ Rovisco Pais, Portugal
}

*Corresponding author: Inês Ferro, Resident in Physical and Rehabilitation Medicine, Centro de Medicina de Reabilitação da Região Centro, Praceta Bernardo Santareno, 4460-262, Senhora da Hora, Rovisco Pais, Portugal, Tel: 919873391

\begin{abstract}
The cancer population is exposed to exceptional factors that might favor sacroiliac joint (SIJ) pain, including the cancer itself and therapy-related side effects. Yet, they are also subject to aging and structural changes. The diagnosis and treatment can be a challenge.

We report a male with SIJ pain and functional repercussion after anal cancer treatment, without signs of recurrence or metastasis. The provocation tests were compatible with sacroiliitis. An ultrasound-guided injection was performed, which significantly relieved his pain and improved functionality.

The International Association for the Study of Pain proposed as diagnostic criteria for SIJ pain: Reproducible pain with provocative tests and complete relieve by local anesthetic infiltration. In this case the etiology is not linear, although the patient only felt this pain after radiation therapy. The SIJ infiltration remains a standard in the diagnostic and therapeutic approach. However, there is no consensus on the optimal modality in cancer patients.
\end{abstract}

\section{Keywords}

Sacroiliitis, Ultrasound injection, Cancer, Pain

\section{Introduction}

Symptomatic SIJ disease is still poorly understood [1]. In the general population, SIJ dysfunction and pain is considered the third most common source of chronic low back pain, after disc disruption and facet joint pathology [2]. In the cancer population, while apparently clinically frequent, it is poorly represented.
Pain may arise from the SIJ itself or from the surrounding structures. Intra-articular pain generators include infection, arthritis, malignancy and spondyloarthropathy. Extra-articular pain generators include fractures, capsular and ligamentous injuries, cysts in the synovium, iliac bone or sacral perineural area, enthesopathy, myofascial pain or hip arthrosis [1]. The age-related degenerative changes (most common in males) only claim clinical relevance when symptomatic.

In cancer population, pain can also result from tumor invasion across the joint or be secondary to the cancer treatment itself. Primary tumors most often reported to invade the SIJ are Ewing and epithelioid sarcoma, osteoid osteoma, osteochondroma, giant cell tumor, sacrococcygeal chordoma, solitary plasmacytoma and myxoid chondrosarcoma, as well as metastases from adenocarcinoma of the prostate, kidney, colon, breast, bladder, lung or rarely others [1].

Nonetheless, frequently no specific cause is identified.

\section{Case Description}

We describe a case of a 72-year-old male who came to an appointment in our Center in December 2019 with a lower back and inguinal pain, and consequent functional limitations. He had a history of a cancer diagnosis in 2015, a moderately differentiated epidermoid carcinoma of the anal canal, classified as

Citation: Ferro I, Sá PC, Pires C, Carvalho F, Constantino J (2021) Ultrasound-Guided Intervention in a Sacroiliitis after Cancer Consequence or Coincidence? A Case Report and Review. Int J Physiatry 7:022. doi.org/10.23937/2572-4215.1510022

Accepted: December 22, 2020: Published: December 24, 2020

Copyright: (c) 2020 Ferro I, et al. This is an open-access article distributed under the terms of the Creative Commons Attribution License, which permits unrestricted use, distribution, and reproduction in any medium, provided the original author and source are credited. 
an cT3NOMO. He was submitted to chemotherapy and radiation that year. Since then, he has been followed by an Oncologist, with no symptoms nor signs of recurrence. His pain complaints started after the radiation cycle, as well as bilateral hip flexion range-ofmotion (ROM) limitation. When questioned, he pointed to the SIJ and the left inguinal regions, claiming the left as the most painful side. It was characterized as a mechanical nociceptive pain, worsened with hip flexion, sitting or walking for a long period of time. He scored the maximum pain intensity as 9/10 (Visual Analogic Scale - VAS) and average of 5-6/10 (VAS). There were no complaints at night, no red flags, no other painful joints, history of enthesitis, dactylitis, or uveitis. There was an insignificant improvement with analgesics or NSAIDs. The functional limitations were the inability to put on socks or shoes, as well as difficulty raising form a sitting position.

Upon observation, there was an active ROM limitation of both hips, with a maximum flexion of $110^{\circ}$ on the right hip and $90^{\circ}$ on the left, with $10^{\circ}$ added in the passive evaluation; The internal rotation was $15^{\circ}$ on the right and $10^{\circ}$ on the left (with borderline pain referred to the SIJ and left groin), with a normal external rotation. There was an intense pain (mimicking "the usual one") with SIJ palpation, especially on the left side. Provocative tests (Gaenslen, Volkmann, Lewin and sacrum compression) were positive, as well as FABER for SIJ pain. There were no other alterations worth mentioning, including in the neurological examination.

The X-ray (Figure 1) shows degenerative changes in both hips and SIJ (with some periarticular sclerosis and blurring of the SIJ margins).

After examination, we considered that the source of most pain was likely the SIJ. We proposed a diagnostic test with ultrasound-guided SIJ injection of an anesthetic, that the patient agreed. The patient was positioned in prone, and with a 1-5 MHz C1-5-RS convex array (from an Logiq ${ }^{\circ}$ P9 ultrasound machine) in a transverse orientation, the lower third of the SIJ was identified; after the aseptic measures, a 20 gauge needle $(0.9 \times 88 \mathrm{~mm})$ was guided to our target to inject $5 \mathrm{ml}$ of $1 \%$ lidocaine. The diagnostic test was positive, with a major improvement in pain (maximum VAS 2/10) and function - minimum pain while putting on his shoes, without help. So, the patient fulfilled the International Association for the Study of Pain (IASP) criteria for the diagnosis of sacroiliitis. Days later, we performed the therapeutic injection with the same methodology, using $4 \mathrm{ml}$ of $1 \%$ lidocaine and $1 \mathrm{ml}$ of methylprednisolone acetate $(40 \mathrm{mg})$, with no complications after the procedure.

Six months later, the patient is still satisfied with the results, with a basal pain scoring in VAS $1 / 10$, with no functional limitations or need of analgesics. There was a slight improvement in the ROM, still painless, with no complaints on palpation or provocative tests.

\section{Discussion}

\section{Symptoms}

The presentation of a sacroiliitis can be very similar to other causes of chronic back pain, which difficult its definitive diagnosis. In cancer patients, underlying pathology can easily overlap the manifestations of SIJ pain. Generally, the pain is described as vague or dull, predominantly felt on one side. It can also produce pelvic pain and stiffness with reduced ROM in the lower back, hips and pelvis, which may hinder position changes, such as standing from a seated position or sitting on a hard surface. The most common referral

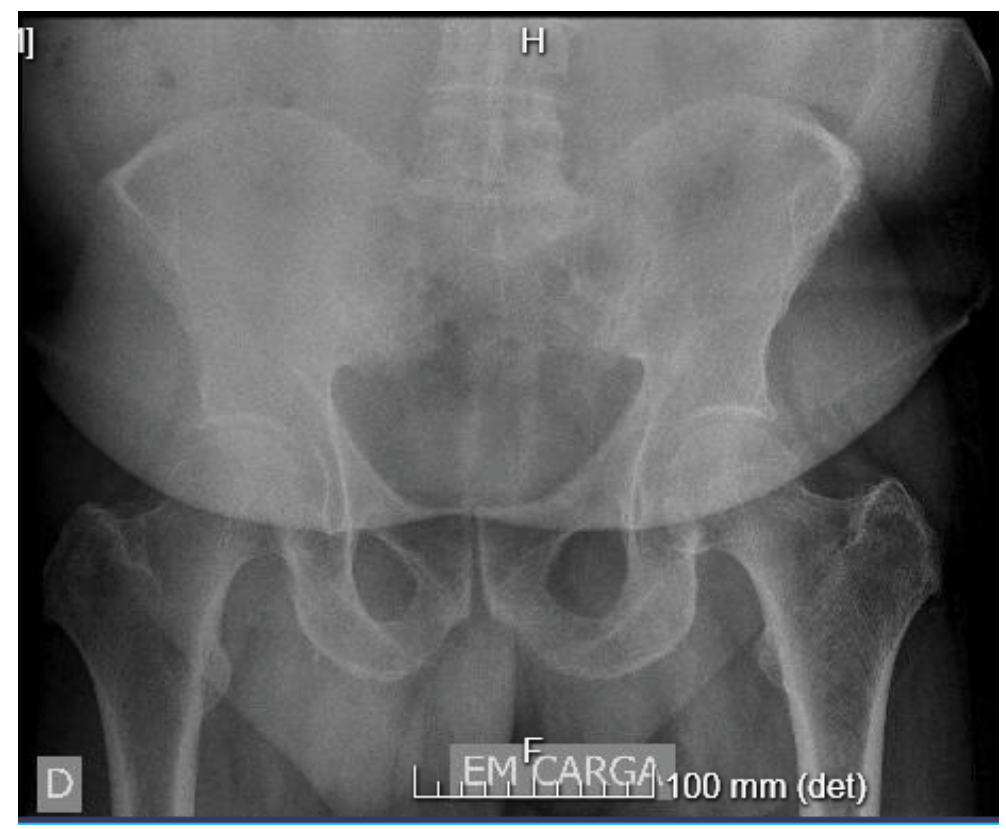

Figure 1: Lumbar and Hip Radiography, from September 2019. 
Table 1: International Association for the Study of Pain criteria for the diagnosis of SIJ pain [4].

1. Pain that is present in the region of the SIJ.

2. Stressing the SI joint by performing clinical tests that are selective for the joint reproduces the patient's pain.

3. Selectively infiltrating the putatively symptomatic joint with local anesthetic completely relieves the patient of pain.

patterns include the gluteal region (94\%) lower lumbar region $(72 \%)$, groin (14\%), upper lumbar region (6\%), and abdomen (2\%) [3].

\section{Diagnostic criteria}

The IASP defines SIJ pain as pain that arises from this anatomical area, but since clinical features alone were unreliable for diagnosis of its source, it has proposed a set of additional criteria that addresses mechanical disorders of the SIJ [4]: Pain should be reproducible by performing specific provocation tests and should be completely relieved by infiltration of the joint with a local anesthetic (Table 1). Three or more provocation tests specific to the SIJ are recommended. In cancer patients, determining the cause of pain should be directed to investigating intra-articular and extra-articular causes, including bone metastasis.

\section{Imaging}

Imaging studies are of limited diagnostic value in SIJ pain, but they should be considered in the setting of "red flags" (fracture, infection, malignant or rheumatologic causes). The initial imaging modality for the diagnostic remains the conventional radiography. A CT scan can be useful for bone-related changes, whereas MRI is the gold standard when considering an inflammatory disorder. Also, some studies suggest that most of those who met the strict diagnostic criteria for SIJ pain had at least one abnormality on their hip radiography [1]. This suggests that clinicians should consider hip pathology in the differential diagnosis.

Our patient had confounding factors for the diagnosis, like the hip flexion and rotation limitation and the inguinal irradiation of pain, that might indicate hip osteoarthritis. However, considering all the findings and IASP criteria, there was a high suspicion of a mainly SIJ source for the complaints, so it was our first goal to prove it by performing a diagnostic test with an ultrasound-guided infiltration.

\section{Intra-articular SIJ injection}

Intra-articular injection of a local anesthetic remains the standard in the diagnosis of SIJ dysfunction. It is considered positive if the pain is relieved by at least $75 \%$ after the procedure [1]. The injector should consider factors that may contraindicate the technique, such as immunosuppression, thrombocytopenia or metastatic disease. SIJ injections require a certain level of expertise. Because of the complex anatomy of the SIJ, injection with a blind palpation technique often results in low accuracy. Therefore, it is important to use image guidance for a successful procedure. Ultrasound-guided injection on the SIJ has been demonstrated to have a high success rate (up to $90 \%$ ) [5]. It is recommended the use of a curvilinear array as it covers a wider cross-section area. For the technique, the patient must be in a prone position, with the transducer in a transverse orientation to identify the sacral hiatus first. After identifying the sacral cornu, the transducer is moved laterally until the lateral edge of the sacrum is observed. The transducer is then moved upwards until the bony contour of the ilium is identified. The cleft between the bony contours of the sacrum and ilium represents the posterior aspect of the SIJ. The lower third of the SIJ can be identified by tilting the transducer in a caudal direction. This is the portion in which the injection should be performed, because of its synovial component, with a medial to lateral approach. The variable joint innervation and failure to infiltrate the entire SIJ are some reasons for inconstant results. Yet, it has been reported that even if the injectant is not administered accurately into the SIJ, ultrasound guidance can at least ensure periarticular deposition of the injectant to the lower third portion, which is believed to be equally effective in improving the pain induced by sacroiliitis [6].

\section{Treatment}

There is no consensus on the ideal therapeutic modality for SIJ syndrome. In the general population, we can consider conservative, interventional or surgical therapies. However, this doesn't apply in the cancer population. Conservative interventions, such as physical therapy and analgesics, have shown limited efficacy in cancer-free patients, but might be the first and only option in cancer patients who are not a candidate for interventional procedures (because of poor general condition, concomitant use of large dose of systemic steroids, immunotherapy, anticoagulation, metastatic disease, thrombocytopenia, neutropenia, etc.) or surgery (because of short life expectancy) [1]. Antiinflammatory drugs may also be useful. Rehabilitation (involving physiotherapy with mobilization activities, strengthening, stretching and proprioceptive training, activity modification, psychology) is a part of a multidisciplinary treatment approach, and can be considered before or in conjunction with invasive procedures. As for the interventional approach, localanesthetic injections or radiofrequency denervation (rhizotomy) have been attempted as therapeutic options. There is some evidence that pulsed radiofrequency denervation of lateral branches from L4-S3 helps to control the pain [7]. However, in noncancer patients it yielded similar results to placebo one month after the 
intervention [8]. Neither clinical recommendations nor studies have addressed the efficacy of rhizotomy on SIJ pain in cancer patients. There is one case report that used pulsed radiofrequency for the treatment of SIJ pain due to local metastases, that reported a $>70 \%$ reduction in pain lasting for 10 months [9]. The International Society for the Advancement of Spine Surgery recommends minimally invasive SIJ fusion for patients who have shown at least $75 \%$ pain reduction after an SIJ injection and in whom conservative treatment or rhizotomy has failed. However, the presence of tumor at the SIJ is a contraindication, and this approach has not been documented in cancer patients.

This clinical case raises some questions: Is the pain consequence or coincidence, connected to the cancer background history? Perhaps the most correct answer is: We cannot be sure. Considering the possible connection to the cancer history, we know that our patient is periodically monitored, and showed no recent signs of recurrence or metastasis. So, and bearing in mind the other findings, the mechanical pain and the absence of red flags, we considered a probable degenerative etiology, knowing we cannot certainly tell if there was an accelerated course caused by the cancer treatments.

As the patient had complaints that could be from hip arthritis or SIJ dysfunction, how do we decide how to proceed? Although our patient showed signs of both, we can differentiate which is the most probable cause of pain through a number of specific maneuvers, imaging and clinical judgement. Most of all, the success of the diagnostic and therapeutic injections allows us to state that the functional repercussion was most likely due to the SIJ pain.

\section{Conclusion}

SIJ dysfunction in cancer patients is a clinical challenge, and the additional risk factors for pain and dysfunction should be considered and actively looked for. The etiological diagnosis may be harder, and should be based on a combination of physical evaluation, considering pain referral patterns, at least 3 provocative tests, imaging and a diagnostic injection. Treatment can be challenging, but there certainly are options.

In this clinical case, the etiology is not linear, although the patient related the onset of pain and functional limitations with the radiation therapy, and there is no evidence of recent recurrence or metastasis. SIJ injection remains the standard for the diagnosis, and it was significantly positive in our patient, improving both pain and function.

This case highlights the importance of a thorough physical examination and a good clinical reasoning. An ultrasound-guided procedure is an effective weapon both in diagnostic and treatment of this nosological entity, improving the intervention success.

\section{Acknowledgments}

The authors disclose any form of conflict of interest.

\section{References}

1. Roldan C, Javed S, Cata J (2020) Sacroiliitis in cancer patients: a review of current literature. Pain Manag 10: 107115.

2. Sembrano JN, Polly DW (2009) How often is low back pain not coming from the back? Spine (Phila Pa 1976) 34: E27-E32.

3. Slipman $\mathrm{C}$, Jackson $\mathrm{H}$, Lipetz J, Chan $\mathrm{K}$, Lenrow D, et al. (2000) Sacroiliac joint pain referral zones. Arch Phys Med Rehabil 81: 334-338.

4. Merskey H, Bogduk N (1994) Classification of chronic pain: Descriptions of chronic pain syndromes and definitions of pain terms. IASP Press WA, USA, 190-191.

5. Klauser A, De Zordo T, Feuchtner G, Sögner P, Schirmer $M$, et al. (2008) Feasibility of ultrasound-guided sacroiliac joint injection considering sonoanatomic landmarks at two different levels in cadavers and patients. Arthritis Rheum 59: 1618-1624.

6. Hartung W, Ross C, Straub R, Feuerbach S, Schölmerich $\mathrm{J}$, et al. (2010) Ultrasound-guided sacroiliac joint injection in patients with established sacroiliitis: Precise IA injection verified by MRI scanning does not predict clinical outcome. Reumatology 49: 1479-1482.

7. Vallejo R, Benyamin R, Stanton G, Joseph N (2006) Pulsed radiofrequency denervation for the treatment of sacroiliac joint syndrome. Pain Med 5: 429-434.

8. Cohen S, Hurley R, Buckenmaier C, Kurihara C, Morlando B, et al. (2008) Randomized placebo-controlled study evaluating lateral branch radiofrequency denervation for sacroiliac joint pain. Anesthesiology 109: 279-288.

9. Yi Y, Lee N, Kwon Y, Jang J, Lim S (2016) Pulsed radiofrequency application for the treatment of pain secondary to sacroiliac joint metastases. Korean J Pain 29: 53-56. 